\title{
PROBLEM ON MINIMAL TYPE OF ENTIRE FUNCTIONS OF ORDER $\rho \in(0,1)$ WITH POSITIVE ZEROES OF PRESCRIBED DENSITIES AND STEP
}

\section{O.V. SHERSTYUKOVA}

Dedicated to the memory of professor

Igor' Fedorovich Krasichkov-Ternovskii

\begin{abstract}
We consider the problem on the least possible type of entire functions of order $\rho \in(0,1)$, whose zeroes lie on a ray and have prescribed densities and step. We prove the sharpness of the estimate obtained previously by the author for the type of these functions. We provide a detailed justification for the construction of the extremal entire function in this problem.
\end{abstract}

Keywords: type of an entire function, upper, lower densities and step of sequence of zeroes, extremal problem.

Mathematics Subject Classification: 30D15

\section{INTRODUCTION}

In work [1] of the author the following problem was formulated. Consider all entire functions whose zeroes are located on a ray and have a prescribed upper and lower density and step with an exponent $\rho \in(0,1)$. We need to find the least possible type for such functions of order $\rho$. In paper [1] the proof of a sharp lower bound for type is provided and an idea of constructing an extremal function was briefly discussed. The present work is a continuation of paper [1] and provides a detailed description of constructing the zero set of an entire function possessing the least possible type. The checking that the constructed example is extremal required cumbersome calculations, many serious efforts and much time. This is the reason why we discuss the construction separately. As a result, the study of the problem formulated in [1] becomes completed.

We introduce needed definition. Given an entire function $f(z)$, its type with order $\rho>0$ is defined by the formula

$$
\sigma_{\rho}(f)=\varlimsup_{r \rightarrow+\infty} r^{-\rho} \ln \max _{|z|=r}|f(z)|
$$

and it is often called $\rho$-type. We assume that function $f(z)$ has infinitely many zeroes. They are taken counting multiplicity in the ascending order of the absolute values and they form the sequence $\Lambda_{f}=\Lambda=\left(\lambda_{n}\right)_{n=1}^{\infty}$. By $n_{\Lambda}(r)=\sum_{\left|\lambda_{n}\right| \leqslant r} 1$ we denote the counting function of sequence $\Lambda$. The upper $\rho$-density of $\Lambda$ is the quantity

$$
\bar{\Delta}_{\rho}(\Lambda)=\varlimsup_{r \rightarrow+\infty} \frac{n_{\Lambda}(r)}{r^{\rho}}=\varlimsup_{n \rightarrow \infty} \frac{n}{\left|\lambda_{n}\right|^{\rho}} .
$$

O.V. Sherstyukova, Problem on minimal type of entire functions of order $\rho \in(0,1)$ With POSITIVE ZEROES OF PRESCRIBED DENSITIES AND STEP.

(C)SHERSTYUKOVA O.V. 2015.

Submitted October 1, 2015. 
The corresponding lower limit is called lower $\rho$-density of $\Lambda$ and is denoted by $\Delta_{\rho}(\Lambda)$. We introduce one more characteristics

$$
h_{\rho}(\Lambda)=\varliminf_{n \rightarrow \infty}\left(\left|\lambda_{n+1}\right|^{\rho}-\left|\lambda_{n}\right|^{\rho}\right)
$$

called $\rho$-step of sequence $\Lambda$. For each sequence $\Lambda$ of a finite $\rho$-density the relation $\bar{\Delta}_{\rho}(\Lambda) h_{\rho}(\Lambda) \leqslant$ 1 holds true.

In what follows we study only entire functions $f(z)$ with the zeros in a ray assuming for the definiteness that all zeroes are positive. We fix both densities and the step of the sequence of zeroes $\Lambda_{f}=\Lambda$ for some exponent $\rho \in(0,1)$. More precisely, we fix numbers $\rho, \beta, \alpha, h$ satisfying the conditions

$$
\rho \in(0,1), \quad \beta>0, \quad \alpha \in[0, \beta], \quad h \in[0,1 / \beta],
$$

and we consider the extremal value

$$
s(\alpha, \beta, h ; \rho) \equiv \inf \left\{\sigma_{\rho}(f): \Lambda_{f} \subset \mathbb{R}_{+}, \bar{\Delta}_{\rho}\left(\Lambda_{f}\right)=\beta, \underline{\Delta}_{\rho}\left(\Lambda_{f}\right) \geqslant \alpha, h_{\rho}\left(\Lambda_{f}\right) \geqslant h\right\} .
$$

For the $\rho$-types of entire functions in Definition (1) the estimate

$$
\sigma_{\rho}(f) \geqslant \frac{\pi \alpha}{\sin \pi \rho}+\sup _{a>0} \varphi(a)
$$

was proved (see [1]), where

$$
\begin{gathered}
\varphi(a)=\varphi_{\alpha, \beta, h, \rho}(a)=\int_{a(\alpha / \beta)^{1 / \rho}}^{a} \frac{\beta a^{-\rho}-\alpha \tau^{-\rho}}{1+\tau} d \tau+\frac{s}{h} \int_{a}^{a \nu^{1 / \rho}} \frac{\nu \tau^{-\rho}-a^{-\rho}}{1+\tau} d \tau, \\
s=1-\beta h \in[0,1], \quad \nu=\frac{1-\alpha h}{1-\beta h} \in[1,+\infty] .
\end{gathered}
$$

As $h=0$ and $h=1 / \beta$, formula (3) should be treated in a certain limiting sense.

As $h=0$, the second term in (3) is equal to

$$
\lim _{h \rightarrow 0} \frac{1}{h} \int_{a}^{a \nu^{1 / \rho}} \frac{\nu \tau^{-\rho}-a^{-\rho}}{1+\tau} d \tau=(\beta-\alpha) \lim _{h \rightarrow 0} \int_{a}^{a \nu^{1 / \rho}} \frac{\tau^{-\rho}}{1+\tau} d \tau=0 .
$$

Here we have applied L'Hôpital rule, Leibnitz formula and the differentiation of an integral w.r.t. a parameter and we have used that $\nu^{1 / \rho} \rightarrow 1$ as $h \rightarrow 0$. Thus, the second term in formula (3) vanishes and estimate (2) gives the sharp result by V.B. Sherstykov in work [2]:

$$
\sigma_{\rho}(f) \geqslant \frac{\pi \alpha}{\sin \pi \rho}+\max _{a>0} \int_{a(\alpha / \beta)^{1 / \rho}}^{a} \frac{\beta a^{-\rho}-\alpha \tau^{-\rho}}{1+\tau} d \tau .
$$

As $h=1 / \beta$, due to the convergence $\nu^{1 / \rho} \rightarrow+\infty$ as $h \rightarrow 1 / \beta$, we evaluate an indeterminate form and obtain that the second term in (3) is equal to

$$
\lim _{h \rightarrow 1 / \beta} \frac{s}{h} \int_{a}^{a \nu^{1 / \rho}} \frac{\nu \tau^{-\rho}-a^{-\rho}}{1+\tau} d \tau=\beta \lim _{h \rightarrow 1 / \beta}(1-\beta h) \int_{a}^{a \nu^{1 / \rho}} \frac{\tau^{-\rho}}{1+\tau} d \tau=(\beta-\alpha) \int_{a}^{+\infty} \frac{\tau^{-\rho}}{1+\tau} d \tau .
$$

Then formula (3) becomes

$$
\varphi(a)=\int_{a(\alpha / \beta)^{1 / \rho}}^{a} \frac{\beta a^{-\rho}-\alpha \tau^{-\rho}}{1+\tau} d \tau+(\beta-\alpha) \int_{a}^{+\infty} \frac{\tau^{-\rho}}{1+\tau} d \tau
$$


that implies

$$
\sup _{a>0} \varphi(a) \geqslant \lim _{a \rightarrow+0} \varphi(a)=\frac{\pi(\beta-\alpha)}{\sin \pi \rho} .
$$

Here estimate (2) yields the inequality

$$
\sigma_{\rho}(f) \geqslant \frac{\pi \beta}{\sin \pi \rho} .
$$

Since the opposite inequality is always true, it leads us to the exact formula

$$
\sigma_{\rho}(f)=\frac{\pi \beta}{\sin \pi \rho} .
$$

The fact that formula (4) is true for each value $\alpha \in[0, \beta]$ provided $h=1 / \beta$ seems to be new. It was believed before that rule (4) provides the type for order $\rho \in(0,1)$ only for an entire function with a measurable sequence of positive zeroes, i.e., such that $\Lambda_{f} \subset \mathbb{R}_{+}$and the limit $\lim _{r \rightarrow+\infty} r^{-\rho} n_{\Lambda}(r)=\beta$ is well-defined.

We note that our interest to problem (1) is motivated by work 3] by A.Yu. Popov, where the issue on the lowest type was studied without taking into consideration the lower density and the step of the sequence of zeroes. The detailed discussion of extremal problem (1) and a survey of previous results can be found in [1].

In the particular case $\alpha=0$, estimate (2) was obtained by the author in [4], and an appropriate example justifying its sharpness was adduced in [5]. The results of [4], [5] show that

$$
s(\beta, h ; \rho):=s(0, \beta, h ; \rho)=h^{-1} \sup _{a>0}\left\{a^{-\rho} \ln \frac{1+a}{\left(1+a s^{-1 / \rho}\right)^{s}}+\int_{a}^{a s^{-1 / \rho}} \frac{\tau^{-\rho}}{1+\tau} d \tau\right\}
$$

for $s=1-\beta h$.

Let us show that in the general case $\alpha \in[0, \beta]$ estimate (2) is attained. At that, the values $\alpha=0, \alpha=\beta, h=0, h=1 / \beta$ can be excluded from the consideration. In this way we shall justify the identity

$$
s(\alpha, \beta, h ; \rho)=\frac{\pi \alpha}{\sin \pi \rho}+\sup _{a>0} \varphi(a)
$$

for function $\varphi(a)$ defined in (3) and it will provide the complete solution to problem (1).

\section{Proof of Sharpness of estimate (2)}

We fix numbers

$$
\rho \in(0,1), \quad \beta>0, \quad \alpha \in(0, \beta), \quad h \in(0,1 / \beta) .
$$

Let us construct a sequence $\Lambda_{0}=\left(\lambda_{n}\right)_{n=1}^{\infty} \subset \mathbb{R}_{+}$so that

$$
\bar{\Delta}_{\rho}\left(\Lambda_{0}\right)=\beta, \quad \underline{\Delta}_{\rho}\left(\Lambda_{0}\right)=\alpha, \quad h_{\rho}\left(\Lambda_{0}\right)=h,
$$

and the $\rho$-type of the associated canonical product

$$
f_{0}(z)=\prod_{n=1}^{\infty}\left(1-\frac{z}{\lambda_{n}}\right), \quad z \in \mathbb{C}
$$

is calculated by the formula

$$
\sigma_{\rho}\left(f_{0}\right)=\frac{\pi \alpha}{\sin \pi \rho}+\max _{a>0} \varphi(a) .
$$

Function $\varphi(a)$ defined by (3) for the parameters

$$
s=1-\beta h \in(0,1), \quad \nu=\frac{1-\alpha h}{1-\beta h} \in(1,+\infty),
$$


is positive and continuous for $a>0$ and

$$
\lim _{a \rightarrow+0} \varphi(a)=\lim _{a \rightarrow+\infty} \varphi(a)=0 .
$$

This is why in (6) we have max instead of sup in (2). We denote by $a_{0}=a_{0}(\alpha, \beta, h, \rho)$ the point of the maximum of $\varphi(a)$ on the ray $a>0$ and we introduce the function

$$
\psi_{0}(t)= \begin{cases}\alpha, & t \in\left[0, \nu^{-1 / \rho} / a_{0}\right] \cup\left[k^{-1 / \rho} / a_{0},+\infty\right) ; \\ h^{-1}\left(1-\frac{s}{\left(a_{0} t\right)^{\rho}}\right), & t \in\left(\nu^{-1 / \rho} / a_{0}, 1 / a_{0}\right] ; \\ \frac{\beta}{\left(a_{0} t\right)^{\rho}}, & t \in\left(1 / a_{0}, k^{-1 / \rho} / a_{0}\right) .\end{cases}
$$

For the sake of convenience we denote

$$
K(t)=\frac{t^{\rho-1}}{1+t}, \quad t \geqslant 0 .
$$

It allows us to rewrite estimate (2) in a more compact form

$$
\sigma_{\rho}(f) \geqslant \int_{0}^{+\infty} \psi_{0}(t) K(t) d t
$$

and formula (6) can be rewritten as

$$
\sigma_{\rho}\left(f_{0}\right)=\int_{0}^{+\infty} \psi_{0}(t) K(t) d t
$$

The passage from (2) to (8) and from (6) to (9) is based on the fact after the change of variable $\tau=1 / t$ in (3) we get

$$
\varphi(a)=\int_{\nu^{-1 / \rho / a}}^{1 / a}\left[h^{-1}\left(1-\frac{s}{(a t)^{\rho}}\right)-\alpha\right] K(t) d t+\int_{1 / a}^{k^{-1 / \rho} / a}\left(\frac{\beta}{(a t)^{\rho}}-\alpha\right) K(t) d t .
$$

Substituting $a=a_{0}$ into (10), we find

$$
\varphi\left(a_{0}\right)=\int_{0}^{+\infty} \psi_{0}(t) K(t) d t-\alpha \int_{0}^{+\infty} K(t) d t=\int_{0}^{+\infty} \psi_{0}(t) K(t) d t-\frac{\pi \alpha}{\sin \pi \rho}
$$

that yields the required formula

$$
\int_{0}^{+\infty} \psi_{0}(t) K(t) d t=\frac{\pi \alpha}{\sin \pi \rho}+\varphi\left(a_{0}\right)
$$

Our aim is to find entire function (5) with property (9).

Following [2], [3], we first define a sequence $\left(m_{n}\right)_{n=1}^{\infty}$ satisfying the condition $m_{n+1}=m_{n}^{4}$, $n \in \mathbb{N}$, and we let $k=\alpha / \beta \in(0,1)$. We observe that for each $p>0$ the relations

$$
\sum_{n=1}^{j-1} m_{n}^{p}=o\left(m_{j}^{p}\right), \quad \sum_{n=j+2}^{\infty} \frac{1}{m_{n}^{p}}=o\left(\frac{1}{m_{j+1}^{p}}\right), \quad j \rightarrow \infty
$$

hold true. We construct the extremal sequence of positive numbers $\Lambda_{0}$ as follows. In the segments $\left[m_{n}, \nu^{-1 / \rho} m_{n}^{2}\right] \cup\left[k^{-1 / \rho} m_{n}^{2}, m_{n+1}\right]$ we choose points $\lambda_{j}$ so that $\lambda_{j}^{\rho}$ form the arithmetic progression with the step $1 / \alpha$. In semi-intervals $\left(\nu^{-1 / \rho} m_{n}^{2}, m_{n}^{2}\right]$ we locate points $\lambda_{j}$ so that 
$\lambda_{j}^{\rho}$ form the arithmetic progression with the step $h$. Intervals $\left(m_{n}^{2}, k^{-1 / \rho} m_{n}^{2}\right)$ are to be free of points $\lambda_{j}$. Introducing the auxiliary sequence $\Omega:=\left(\lambda_{n}^{\rho}\right)_{n=1}^{\infty}$, for each $n \in \mathbb{N}$ we have

$$
\begin{aligned}
& \Omega \cap\left[m_{n}^{\rho}, \nu^{-1} m_{n}^{2 \rho}\right]=\left\{m_{n}^{\rho}+j / \alpha: \quad j \in \mathbb{N}, \quad j \leqslant \alpha\left(\nu^{-1} m_{n}^{2 \rho}-m_{n}^{\rho}\right)\right\} ; \\
& \Omega \cap\left(\nu^{-1} m_{n}^{2 \rho}, m_{n}^{2 \rho}\right]=\left\{\nu^{-1} m_{n}^{\rho}+j h: \quad j \in \mathbb{N}, \quad j \leqslant h^{-1}\left(1-\nu^{-1}\right) m_{n}^{2 \rho}\right\} ; \\
& \Omega \cap\left(m_{n}^{2 \rho}, k^{-1} m_{n}^{2 \rho}\right)=\emptyset ; \\
& \Omega \cap\left[k^{-1} m_{n}^{2 \rho}, m_{n+1}^{\rho}\right]=\left\{k^{-1} m_{n}^{2 \rho}+j / \alpha: \quad j \in \mathbb{N}, \quad j \leqslant \alpha\left(m_{n+1}^{\rho}-k^{-1} m_{n}^{2 \rho}\right)\right\} .
\end{aligned}
$$

By the definition we have

$$
h_{\rho}\left(\Lambda_{0}\right)=\varliminf_{n \rightarrow \infty}\left(\lambda_{n+1}^{\rho}-\lambda_{n}^{\rho}\right)=h
$$

since $h<1 / \beta<1 / \alpha$.

To calculate $\rho$-densities of sequence $\Lambda_{0}$, we shall need the expressions for counting function $n_{\Omega}(r)$ on various segments of the positive half-line. First of all we note that

$$
\begin{aligned}
n_{\Omega}\left(m_{n}^{\rho}\right) & =\sum_{j=1}^{n-1}\left(\alpha\left(\nu^{-1} m_{j}^{2 \rho}-m_{j}^{\rho}\right)+h^{-1}\left(1-\nu^{-1}\right) m_{j}^{2 \rho}\right)+\sum_{j=2}^{n} \alpha\left(m_{j}^{\rho}-k^{-1} m_{j-1}^{2 \rho}\right)+O(n) \\
& =\alpha\left(m_{n}^{\rho}-m_{1}^{\rho}\right)+\sum_{j=1}^{n-1}\left[\alpha\left(\nu^{-1}-k^{-1}\right)+h^{-1}\left(1-\nu^{-1}\right)\right] m_{j}^{2 \rho}+O(n) .
\end{aligned}
$$

The expression in square brackets vanishes. Indeed,

$\alpha\left(\nu^{-1}-k^{-1}\right)+h^{-1}\left(1-\nu^{-1}\right)=\nu^{-1}\left(\alpha-h^{-1}\right)+h^{-1}-\beta=\frac{1-\beta h}{1-\alpha h} \frac{\alpha h-1}{h}+\frac{1-\beta h}{h}=0$.

This is why the relation

$$
n_{\Omega}\left(m_{n}^{\rho}\right)=\alpha m_{n}^{\rho}+O(n), \quad n \rightarrow \infty,
$$

holds true. If $r \in\left[m_{n}^{\rho}, \nu^{-1} m_{n}^{2 \rho}\right]$, then

$$
n_{\Omega}(r)=n_{\Omega}\left(m_{n}^{\rho}\right)+\alpha\left(r-m_{n}^{\rho}\right)+O(1)=\alpha r+O(n) .
$$

If $r \in\left(\nu^{-1} m_{n}^{2 \rho}, m_{n}^{2 \rho}\right]$, then

$$
\begin{aligned}
n_{\Omega}(r) & =n_{\Omega}\left(\nu^{-1} m_{n}^{2 \rho}\right)+h^{-1}\left(r-\nu^{-1} m_{n}^{2 \rho}\right)+O(1)=\alpha \nu^{-1} m_{n}^{2 \rho}+O(n)+h^{-1}\left(r-\nu^{-1} m_{n}^{2 \rho}\right) \\
& =h^{-1} r+\nu^{-1}\left(\alpha-h^{-1}\right) m_{n}^{2 \rho}+O(n)=h^{-1} r-h^{-1} s m_{n}^{2 \rho}+O(n)=h^{-1}\left(r-s m_{n}^{2 \rho}\right)+O(n) .
\end{aligned}
$$

If $r \in\left(m_{n}^{2 \rho}, k^{-1} m_{n}^{2 \rho}\right)$, then

$$
n_{\Omega}(r)=n_{\Omega}\left(m_{n}^{2 \rho}\right)=h^{-1}\left(m_{n}^{2 \rho}-s m_{n}^{2 \rho}\right)+O(n)=h^{-1}(1-s) m_{n}^{2 \rho}+O(n)=\beta m_{n}^{2 \rho}+O(n) .
$$

If $r \in\left[k^{-1} m_{n}^{2 \rho}, m_{n+1}^{\rho}\right]$, the

$$
n_{\Omega}(r)=n_{\Omega}\left(k^{-1} m_{n}^{2 \rho}\right)+\alpha\left(r-k^{-1} m_{n}^{2 \rho}\right)+O(1)=\beta m_{n}^{2 \rho}+O(n)+\alpha\left(r-k^{-1} m_{n}^{2 \rho}\right)=\alpha r+O(n) .
$$

Finally, for each $n \in \mathbb{N}$ we have the representation (with error terms $O(n)$ as $n \rightarrow \infty$ ):

$$
n_{\Omega}(r)= \begin{cases}\alpha r+O(n), & r \in\left[m_{n}^{\rho}, \nu^{-1} m_{n}^{2 \rho}\right] \cup\left[k^{-1} m_{n}^{2 \rho}, m_{n+1}^{\rho}\right] ; \\ h^{-1}\left(r-s m_{n}^{2 \rho}\right)+O(n), & r \in\left(\nu^{-1} m_{n}^{2 \rho}, m_{n}^{2 \rho}\right] ; \\ \beta m_{n}^{2 \rho}+O(n), & r \in\left(m_{n}^{2 \rho}, k^{-1} m_{n}^{2 \rho}\right) .\end{cases}
$$

Hence, due to the relation $n_{\Lambda_{0}}(r)=n_{\Omega}\left(r^{\rho}\right)$, we find that

$$
\bar{\Delta}_{\rho}\left(\Lambda_{0}\right)=\varlimsup_{r \rightarrow+\infty} \frac{n_{\Omega}(r)}{r}=\beta, \quad \triangleq_{\rho}\left(\Lambda_{0}\right)=\varlimsup_{r \rightarrow+\infty} \frac{n_{\Omega}(r)}{r}=\alpha .
$$


For a fixed $r>0$ we consider the function $\varphi_{r}(t)$ defined by the rule

$$
\varphi_{r}(t):=\frac{n_{\Lambda_{0}}(r t)}{(r t)^{\rho}}=\frac{n_{\Omega}\left((r t)^{\rho}\right)}{(r t)^{\rho}}, \quad t>0 .
$$

It allows us to write the representation for each $n \in \mathbb{N}$ :

$$
\varphi_{r}(t)= \begin{cases}\alpha+O\left(\frac{n}{(r t)^{\rho}}\right), & t \in\left[\frac{m_{n}}{r}, \nu^{-1 / \rho} \frac{m_{n}^{2}}{r}\right] \cup\left[k^{-1 / \rho} \frac{m_{n}^{2}}{r}, \frac{m_{n+1}}{r}\right] ; \\ h^{-1}\left(1-s\left(\frac{m_{n}^{2}}{r t}\right)^{\rho}\right)+O\left(\frac{n}{(r t)^{\rho}}\right), & t \in\left(\nu^{-1 / \rho} \frac{m_{n}^{2}}{r}, \frac{m_{n}^{2}}{r}\right] ; \\ \beta\left(\frac{m_{n}^{2}}{r t}\right)^{\rho}+O\left(\frac{n}{(r t)^{\rho}}\right), & t \in\left(\frac{m_{n}^{2}}{r}, k^{-1 / \rho} \frac{m_{n}^{2}}{r}\right) .\end{cases}
$$

We introduce the function $\Phi_{r}(t)$ depending on a parameter $\left.r>0\right)$ and defined for $t \geqslant 0$ such that

$$
\Phi_{r}(t)=\alpha, \quad t \in\left[0, m_{1} / r\right]
$$

while its restrictions on the segments $\left[m_{n} / r, m_{n+1} / r\right], n \in \mathbb{N}$, read as

$$
\Phi_{r}(t)=\left\{\begin{array}{rr}
\alpha, \quad t \in\left[\frac{m_{n}}{r}, \nu^{-1 / \rho} \frac{m_{n}^{2}}{r}\right] \cup\left[k^{-1 / \rho} \frac{m_{n}^{2}}{r}, \frac{m_{n+1}}{r}\right] ; \\
h^{-1}\left(1-s\left(\frac{m_{n}^{2}}{r t}\right)^{\rho}\right), & t \in\left(\nu^{-1 / \rho} \frac{m_{n}^{2}}{r}, \frac{m_{n}^{2}}{r}\right] ; \\
\beta\left(\frac{m_{n}^{2}}{r t}\right)^{\rho}, & t \in\left(\frac{m_{n}^{2}}{r}, k^{-1 / \rho} \frac{m_{n}^{2}}{r}\right) .
\end{array}\right.
$$

In view of (7), $\rho$-type of entire function (5) can be found by the formula

$$
\sigma_{\rho}\left(f_{0}\right)=\varlimsup_{r \rightarrow+\infty} \int_{0}^{+\infty} \varphi_{r}(t) K(t) d t=\varlimsup_{r \rightarrow+\infty} \int_{0}^{+\infty} \Phi_{r}(t) K(t) d t
$$

since the error terms in the expression for $\varphi_{r}(t)$ makes no influence on $\sigma_{\rho}\left(f_{0}\right)$ (see [2]). Thanks to general estimate (8), in order to prove identity (9), it is sufficient to establish

$$
\sigma_{\rho}\left(f_{0}\right) \leqslant \int_{0}^{+\infty} \psi_{0}(t) K(t) d t
$$

which is implied (see (13)) by the relation

$$
\varlimsup_{r \rightarrow+\infty} \int_{0}^{+\infty}\left(\Phi_{r}(t)-\psi_{0}(t)\right) K(t) d t \leqslant 0 .
$$

So, we need to check (14). First we observe that the formula

$$
\int_{0}^{+\infty}\left(\Phi_{r}(t)-\psi_{0}(t)\right) K(t) d t=\sum_{n=1}^{\infty} \varphi\left(\frac{a_{0} r}{r_{n}}\right)-\varphi\left(a_{0}\right), \quad r>0
$$


holds true, where $r_{n}=a_{0} m_{n}^{2}, n \in \mathbb{N}$. Indeed, employing the definition of function $\Phi_{r}(t)$ and representation (10) for function $\varphi(a)$, we write

$$
\begin{aligned}
& \int_{0}^{+\infty} \Phi_{r}(t) K(t) d t=\alpha \int_{0}^{+\infty} K(t) d t \\
& \quad+\sum_{n=1}^{\infty}\left\{\int_{\frac{m_{n}^{2}}{\nu^{1 / \rho_{r}}}}^{\frac{m_{n}^{2}}{r}}\left[h^{-1}\left(1-s\left(\frac{m_{n}^{2}}{r t}\right)^{\rho}\right)-\alpha\right] K(t) d t+\int_{\frac{m_{n}^{2}}{r}}^{\frac{m_{n}^{2}}{k^{1 / \rho_{r}}}}\left[\beta\left(\frac{m_{n}^{2}}{r t}\right)^{\rho}-\alpha\right] K(t) d t\right\} \\
& =\frac{\pi \alpha}{\sin \pi \rho}+\sum_{n=1}^{\infty} \varphi\left(\frac{r}{m_{n}^{2}}\right) .
\end{aligned}
$$

Thus,

$$
\int_{0}^{+\infty} \Phi_{r}(t) K(t) d t=\frac{\pi \alpha}{\sin \pi \rho}+\sum_{n=1}^{\infty} \varphi\left(\frac{r}{m_{n}^{2}}\right), \quad r>0 .
$$

Deducting identity (11), we arrive at formula (15).

We employ (15) to prove (14). We fix an index $j \geqslant 2$ and we split the expression in the right hand side in (15) into three sums:

$$
\sum_{n=1}^{j-1} \varphi\left(\frac{a_{0} r}{r_{n}}\right), \quad \sum_{n=j+2}^{\infty} \varphi\left(\frac{a_{0} r}{r_{n}}\right), \quad \varphi\left(\frac{a_{0} r}{r_{j}}\right)+\varphi\left(\frac{a_{0} r}{r_{j+1}}\right)-\varphi\left(a_{0}\right) .
$$

Let us estimate them separately for $r \in\left[r_{j}, r_{j+1}\right]$.

We begin with the first sum. Letting $a=a_{0} r / r_{n}$ in representation (10) and applying obvious inequality $K(t) \leqslant t^{\rho-1}$, we obtain

$$
\begin{aligned}
\varphi\left(\frac{a_{0} r}{r_{n}}\right) \leqslant & \int_{\nu^{-1 / \rho} \frac{r_{n}}{a_{0} r}}^{\frac{r_{n}}{a_{0} r}}\left[h^{-1}\left(1-s\left(\frac{r_{n}}{a_{0} r t}\right)^{\rho}\right)-\alpha\right] t^{\rho-1} d t+\int_{\frac{r_{n}}{a_{0} r}}^{k^{-1 / \rho} \frac{r_{n}}{a_{0} r}}\left[\beta\left(\frac{r_{n}}{a_{0} r t}\right)^{\rho}-\alpha\right] t^{\rho-1} d t \\
= & \frac{h^{-1}-\alpha}{\rho}\left(1-\nu^{-1}\right)\left(\frac{r_{n}}{a_{0} r}\right)^{\rho}-h^{-1} s \ln \nu^{1 / \rho}\left(\frac{r_{n}}{a_{0} r}\right)^{\rho}+\beta \ln k^{-1 / \rho}\left(\frac{r_{n}}{a_{0} r}\right)^{\rho} \\
& -\frac{\alpha}{\rho}\left(k^{-1}-1\right)\left(\frac{r_{n}}{a_{0} r}\right)^{\rho} \\
= & \frac{1}{\rho}\left(\frac{r_{n}}{a_{0} r}\right)^{\rho}\left[\frac{1-\alpha h}{h}\left(1-\frac{1-\beta h}{1-\alpha h}\right)-\frac{s}{h} \ln \nu-\beta \ln k-(\beta-\alpha)\right]=A\left(\frac{m_{n}^{2}}{r}\right)^{\rho}
\end{aligned}
$$

with the constant

$$
A=-\frac{1}{\rho h}[s \ln \nu+\beta h \ln k]>0 .
$$

Hence, for $r \geqslant r_{j}=a_{0} m_{j}^{2}$ we have

$$
0<\sum_{n=1}^{j-1} \varphi\left(\frac{a_{0} r}{r_{n}}\right) \leqslant \frac{A}{r^{\rho}} \sum_{n=1}^{j-1} m_{n}^{2 \rho} \leqslant \frac{A}{a_{0}^{\rho}} \frac{1}{m_{j}^{2 \rho}} \sum_{n=1}^{j-1} m_{n}^{2 \rho} .
$$

Applying the former formula in (12) for $p=2 \rho$, we arrive at the relation

$$
\sup _{r \in\left[r_{j}, r_{j+1}\right]} \sum_{n=1}^{j-1} \varphi\left(\frac{a_{0} r}{r_{n}}\right) \rightarrow 0, \quad j \rightarrow \infty .
$$


We proceed to the second sum. Letting once again $a=a_{0} r / r_{n}$ in representation (10) and applying another obvious inequality $K(t) \leqslant t^{\rho-2}$, we obtain

$$
\begin{aligned}
\varphi\left(\frac{a_{0} r}{r_{n}}\right) \leqslant & \int_{\nu^{-1 / \rho \frac{r_{n}}{a_{0} r}}}^{\frac{r_{n}}{a_{0} r}}\left[h^{-1}\left(1-s\left(\frac{r_{n}}{a_{0} r t}\right)^{\rho}\right)-\alpha\right] t^{\rho-2} d t+\int_{\frac{r n}{a_{0} r}}^{k^{-1 / \rho} \frac{r_{n}}{a_{0} r}}\left[\beta\left(\frac{r_{n}}{a_{0} r t}\right)^{\rho}-\alpha\right] t^{\rho-2} d t \\
= & \frac{h^{-1}-\alpha}{\rho-1}\left(1-\nu^{1 / \rho-1}\right)\left(\frac{r_{n}}{a_{0} r}\right)^{\rho-1}-h^{-1} s\left(\nu^{1 / \rho}-1\right)\left(\frac{r_{n}}{a_{0} r}\right)^{\rho-1} \\
& +\beta\left(1-k^{1 / \rho}\right)\left(\frac{r_{n}}{a_{0} r}\right)^{\rho-1}+\frac{\alpha}{1-\rho}\left(k^{1 / \rho-1}-1\right)\left(\frac{r_{n}}{a_{0} r}\right)^{\rho-1}=B\left(\frac{m_{n}^{2}}{r}\right)^{\rho-1}
\end{aligned}
$$

with the constant

$$
B=\frac{\left(h^{-1}-\alpha\right)\left(\nu^{1 / \rho-1}-1\right)-\alpha\left(1-k^{1 / \rho-1}\right)}{1-\rho}-h^{-1} s\left(\nu^{1 / \rho}-1\right)+\beta\left(1-k^{1 / \rho}\right)>0 .
$$

Hence, for $r \leqslant r_{j+1}=a_{0} m_{j+1}^{2}$ we have

$$
0<\sum_{n=j+2}^{\infty} \varphi\left(\frac{a_{0} r}{r_{n}}\right) \leqslant B r^{1-\rho} \sum_{n=j+2}^{\infty} \frac{1}{m_{n}^{2(1-\rho)}} \leqslant B a_{0}^{1-\rho} m_{j+1}^{2(1-\rho)} \sum_{n=j+2}^{\infty} \frac{1}{m_{n}^{2(1-\rho)}} .
$$

Applying the latter formula in $(12)$ for $p=2(1-\rho)$, we arrive at the relation

$$
\sup _{r \in\left[r_{j}, r_{j+1}\right]} \sum_{n=j+2}^{\infty} \varphi\left(\frac{a_{0} r}{r_{n}}\right) \rightarrow 0, \quad j \rightarrow \infty .
$$

Let us estimate the third sum. In the estimates we make use of the fact that $a_{0}$ is the maximum point of a positive continuous for $a>0$ function $\varphi(a)$, while the function vanishes at zero and at infinity. We consider two cases: $r \in\left[r_{j}, \sqrt{r_{j} r_{j+1}}\right]$ and $r \in\left[\sqrt{r_{j} r_{j+1}}, r_{j+1}\right]$.

In the first case we have $r_{j} / r_{j+1} \leqslant r / r_{j+1} \leqslant \sqrt{r_{j} / r_{j+1}}$. This is why the right hand in the estimate

$$
\varphi\left(\frac{a_{0} r}{r_{j}}\right)+\varphi\left(\frac{a_{0} r}{r_{j+1}}\right)-\varphi\left(a_{0}\right) \leqslant \varphi\left(\frac{a_{0} r}{r_{j+1}}\right)
$$

tends to zero as $j \rightarrow \infty$ uniformly in $r \in\left[r_{j}, \sqrt{r_{j} r_{j+1}}\right]$.

In the second case we have $\sqrt{r_{j+1} / r_{j}} \leqslant r / r_{j} \leqslant r_{j+1} / r_{j}$. This is why the right hand side in the estimate

$$
\varphi\left(\frac{a_{0} r}{r_{j}}\right)+\varphi\left(\frac{a_{0} r}{r_{j+1}}\right)-\varphi\left(a_{0}\right) \leqslant \varphi\left(\frac{a_{0} r}{r_{j}}\right)
$$

again tends to zero as $j \rightarrow \infty$ uniformly in $r \in\left[\sqrt{r_{j} r_{j+1}}, r_{j+1}\right]$.

Finally,

$$
\varlimsup_{j \rightarrow \infty} \sup _{r \in\left[r_{j}, r_{j+1}\right]}\left\{\varphi\left(\frac{a_{0} r}{r_{j}}\right)+\varphi\left(\frac{a_{0} r}{r_{j+1}}\right)-\varphi\left(a_{0}\right)\right\} \leqslant 0 .
$$

Combining (15)-(18), we obtain (14). Thus, function (5) satisfies (9). The example of an extremal function is constructed. Together with the result of work [1] it proves the following statement.

Theorem. For each $\rho \in(0,1), \beta>0, \alpha \in[0, \beta], h \in[0,1 / \beta]$, extremal value (1) is calculated by the formula

$$
s(\alpha, \beta, h ; \rho)=\frac{\pi \alpha}{\sin \pi \rho}+\sup _{a>0} \varphi(a)
$$


where function $\varphi(a)$ is defined as

$$
\varphi(a)=\int_{a(\alpha / \beta)^{1 / \rho}}^{a} \frac{\beta a^{-\rho}-\alpha \tau^{-\rho}}{1+\tau} d \tau+\frac{s}{h} \int_{a}^{a \nu^{1 / \rho}} \frac{\nu \tau^{-\rho}-a^{-\rho}}{1+\tau} d \tau, \quad s=1-\beta h, \quad \nu=\frac{1-\alpha h}{1-\beta h} .
$$

The infimum $s(\alpha, \beta, h ; \rho)$ is attained at an entire function with a sequence of positive simple zeroes having $\rho$-densities $\bar{\Delta}_{\rho}(\Lambda)=\beta, \Delta_{\rho}(\Lambda)=\alpha$ and $\rho$-step $h_{\rho}(\Lambda)=h$.

\section{BIBLIOGRAPHY}

1. O.V. Sherstyukova. On extremal type of an entire function of order less than unity with zeros of prescribed densities and step // Ufimskij Matem. Zhurn. 4:1, 161-165 (2012). [Ufa Math. J. 4:1, 151-155 (2012).]

2. G.G. Braichev, V.B. Sherstyukov. On the least possible type of entire functions of order $\rho \in(0,1)$ with positive zeros // Izv. RAN. Ser. Matem. 75:1, 3-28 (2011). [Izv. Math. 75:1, 1-27 (2011).]

3. A.Yu. Popov. The least possible type under the order $\rho<1$ of canonical products with positive zeros of a given upper $\rho$-density // Vestn. Mosk. Univ. Ser. 1. Matem. Mekh. 1, 31-36 (2005). [Mosc. Univ. Math. Bull. 60:1, 32-36 (2005).]

4. O.V. Sherstyukova. On influence of the step of a sequence of zeroes of an entire function of the order less than one for its type // Nauka v VUZakh. Matem. Inform. Fiz. Obraz. 192-195 (2010). (in Russian).

5. O.V. Sherstyukova. On the least type of entire functions of order $\rho \in(0,1)$ with zeroes in a ray // Izv. Saratov. Univ. Nov. Ser. Matem. Mekh. Inform. 4, 433-441. (2015). (in Russian).

Ol'ga Vladimirovna Sherstyukova,

Moscow State Pedagogical University,

M. Pirogovskaya str., 1,

199296, Moscow, Russia

E-mail: sherov73@mail.ru 